International Journal of Pure and Applied Mathematics

Volume 105 No. 3 2015, 427-430

ISSN: 1311-8080 (printed version); ISSN: 1314-3395 (on-line version)

url: http://www.ijpam.eu

doi: http://dx.doi.org/10.12732/ijpam.v105i3.10

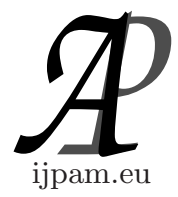

\title{
SINGULAR INNER FUNCTION AND ANALYTIC BOUNDED POINT EVALUATION
}

\author{
Kifah Al-Hami \\ Mathematics Department \\ College of Science \\ University of Bahrain, 32038, BAHRAIN
}

\begin{abstract}
In Section 1, I constructed a singular inner function which is an upper bound for a given outer function, i.e. replacing an outer function with a constant multiple of a singular inner

In Section 2, I proved how unit disk containment is not effected for the analytic bounded point evaluation by a restricted measure.
\end{abstract}

AMS Subject Classification: 53C20, 53B20

Key Words: outer function, singular inner function, Carleson set, bounded point evaluation

\section{Construction of a Singular Inner Function from a Given Outer One}

Lets start first by introducing the following three definitions :

Definition 1.1. A function $F$ defined and analytic in $D$ is called an outer function if it has the form

$$
F(z)=\chi \exp \left[\frac{1}{2 \pi} \int_{0}^{2 \pi} \frac{e^{i t}+z}{e^{i t}-z} \log k(t) d t\right] \quad(z \in D),
$$

where $k$ is a non-negative Lebesgue measurable function on $\partial D, \log (k(t)) \in$ $L^{1}(m)$ and $\chi$ is a complex number of modulus 1

Received: September 14, 2015

(C) 2015 Academic Publications, Ltd. url: www.acadpubl.eu 
Definition 1.2. A function $S_{\mu}$ defined and analytic in $D$ is called a singular inner function if it has the form

$$
S_{\mu}(z)=\exp \left[-\int_{\partial D} \frac{\xi+z}{\xi-z} d \mu(z)\right] \quad(z \in D)
$$

where $\mu$ is a finite non-negative Borel measure on $\partial D$ that is singular with respect to $m$

Definition 1.3. A subset $E$ of $\partial D$ is called a Carleson set if:

1) $E$ is closed;

2) $E$ is of Lebesgue measure zero ; $|E|=0$;

3) $\sum_{\nu}\left|I_{\nu}\right| \log \frac{1}{\left|I_{\nu}\right|}<\infty$, where $\left\{I_{\nu}\right\}$ are the complementary arcs of $E$

Now, the following Theorem which appears in [1] is of great importance for the Theorem that follows

Theorem 1.4. Let $\Omega$ be any Jordan domain in $D$, with $m(\partial D \bigcap \partial \Omega)=0$, let $0 \leq h \in L^{1}(d m)$ and let $P_{z}(\zeta)$ be the Poisson kernal on $\partial D$ evaluated at $z$ in $\Omega$. Then, for any $\epsilon>0$, there exists a finite measure $\mu$ with support in $\partial D$ such that $\mu \perp m$, and $\mu(E)=0$ for every Carleson set $E$ in $\partial D$ and $\left|\int_{\partial D}\left(P_{z}(\zeta) d \mu-P_{z}(\zeta) h d m\right)\right| \leq \epsilon$ for all $z$ in $\Omega$.

In this Theorem we construct a special singular inner function which is an upper bound for a given outer function

Theorem 1.5. Let $\Omega$ be any Jordan domain in $D$, with $m(\partial D \bigcap \partial \Omega)=0$ and let $F$ be an outer function on $D ; F \neq 0$. Then there exists a singular inner function $S_{\mu}$, with $\mu(E)=0$ for every Carleson set $E$ in $\partial D$, such that $\left|S_{\mu}(z)\right| \leq 3 .|F(z)|$ for all $z$ in $\Omega$.

Proof. Since $F$ is an outer function, $|F(z)|=e^{\int_{\partial D} P_{z}(\zeta) \log h(\zeta) d m(\zeta)}$ without loss of generality we can assume that $0 \leq h \leq 1$ on $\partial D$. Now, by Theorem 1.4 , there exists a finite positive Borel measure $\mu$ such that $\mu \perp m, \mu(E)=0$ for every Carleson set $E \subseteq \partial D$ and $\left.\mid \int_{\partial D}\left(P_{z}(\zeta) d \mu(\zeta)-P_{z}(\zeta)\right) \log h(\zeta) d m(\zeta)\right) \mid \leq 1$ for all $z$ in $\Omega$. Let $S_{\mu}$ be the corresponding singular inner function. Then $\left|S_{\mu}(z)\right|=$ $e^{-\int_{\partial D} P_{z}(\zeta) d \mu(\zeta)}$ and therefore, $\left|S_{\mu}(z) / F(z)\right|=e^{-\int_{\partial D}\left(P_{z}(\zeta) d \mu-\int_{\partial D} P_{z}(\zeta) \log (h) d m\right)} \leq$ $e^{\int_{\partial D}\left(P_{z}(\zeta)|\log (h)| d m-P_{z}(\zeta) d \mu\right)} \leq 3$ for all $z$ in $\Omega$. 


\section{The set of Overconvergence abpe $\left(P^{t}(\mu)\right)$}

We now turn to the topic of point evaluations and establish some "overconvergence" results

With $\mu$ and $P^{t}(\mu)$ as before, a complex number $z$ is called a bounded point evaluation for $P^{t}(\mu)$ if there is a constant $M$ such that $|p(z)| \leq M$. ||$p \|_{L^{t}(\mu)}$ for all polynomials $p$; the collection of all such points is denoted $b p e\left(P^{t}(\mu)\right)$. If $z \in C$ and there are positive constants $M$ and $r$ such that $|p(w)| \leq M \cdot|| p \|_{L^{t}(\mu)}$ whenever $p$ is a polynomial and $|w-z|<r$, then we call $z$ an analytic bounded point evaluation for $P^{t}(\mu)$; the set of all points $z$ of this type is denoted by abpe $\left(P^{t}(\mu)\right)$. Notice that abpe $\left(P^{t}(\mu)\right)$ is an open subset of bpe $\left(P^{t}(\mu)\right)$ and, by the Maximum Modulus Theorem, each component of abpe $\left(P^{t}(\mu)\right)$ is simply connected. If $z \in \operatorname{abpe}\left(P^{t}(\mu)\right)$, then by the Hahn-Banach and Riesz Representation Theorems, there exists $K$, in $L^{s}(\mu)(1 / s+1 / t=1)$ such that $p(z)=\int p(\xi) K_{z}(\xi) d \mu(\xi)$ for each polynomial $p$. For $f$ in $P^{t}(\mu)$, define $\hat{f}$ on bpe $\left(P^{t}(\mu)\right)$ by $\hat{f}(z)=\int f(\xi) K_{z}(\xi) d \mu(\xi)$. Observe that $\hat{f}=$ fa.e. $\mu$ on bpe $\left(P^{t}(\mu)\right)$ and in fact $z \mapsto \hat{f}(z)$ is analytic on abpe $\left(P^{t}(\mu)\right)$. The set abpe $\left(P^{t}(\mu)\right)$ support $(\mu)$ can be thought of as a set of overconvergence for $P^{t}(\mu)$. We now establish two results that address this topic of overconvergence.

Lemma 2.1. Let $\mu$ be a finite, positive Borel measure with compact support in $C$. Let $K$ be a compact subset of abpe $\left(P^{t}(\mu)\right)$ and let $M=$ $\sup \left\{|p(z)|^{t}: z \in K\right\}, p \in P$ and $\|p\|_{L^{t}(\mu)}^{t}=1$. If $\mu(K)<1 / M$, then $\|p\|_{L^{t}(\mu)} \leq 1 /(1-M . \mu(K))^{1 / t} \cdot\|P\|_{L^{t}\left(\mu_{(C / K)}\right)}$ for every polynomial $p$.

Proof. By the definition of $M,|p(z)|^{t} \leq M .\|p\|_{L^{t}(\mu)}^{t}$ for every polynomial $p$. Therefore, $\int_{C}|p|^{t} d \mu=\int_{C / K}|P|^{t} d \mu+\int_{K}|p|^{t} d \mu \leq \int_{C / K}|p|^{t} d \mu+M .\left.|| p\right|_{L^{t}(\mu)} ^{t} \mu(K)$, and hence $(1-M \cdot \mu(K)) \cdot \int_{C}|p|^{t} d \mu \leq \int_{C / K}|p|^{t} d \mu$. Consequently, $\int_{C}|p|^{t} d \mu \leq$ $\frac{1}{(1-M . \mu(K))} \int_{C / K}|p|^{t} d \mu$

Proposition 2.2. Let $\mu$ be a finite, positive Borel measure with compact support in $C$ such that $D \subseteq$ abpe $\left(P^{t}(\mu)\right)$. If $K$ is a compact subset of $D$, then $D \subseteq \operatorname{abpe}\left(P^{t}\left(\mu_{(C / K)}\right)\right)$

Proof. Let $\rho=\max \{|z|: z \in K\}$. Now choose $r$ such that $\rho \leq r \leq 1$. Since $\{z:|z| \leq r\}$ is a compact subset of $\operatorname{abpe}\left(P^{t}(\mu)\right)$, there exists $M_{r}>1$ such that $|p(z)| \leq M_{r} \cdot|| p \|_{L^{t}(\mu)}$ whenever $|z| \leq r$ and $p \in P$. Let $f_{n}(z)=$ $\left(\frac{z}{r}\right)^{n}$. Now, if $|z|=r$ and $p$ is a polynomial, then $|p(z)|=\left|p(z) \cdot f_{n}(z)\right| \leq$ $M_{r}\left\|p f_{n}\right\|_{L^{t}(\mu)}=M_{r}\|p\|_{L^{t}\left(\mu_{n}\right)}$ ( where $\left.d \mu_{n}=\left|f_{n}\right|^{t} d \mu\right)$. Since $f_{n} \rightarrow o$ uniformly on $K$, there exists $N$ such that $\mu_{N}(K) \cdot M_{r} \leq 1 / 2^{t}$. So, by Lemma $2 \cdot 1,|p(z)| \leq$ 
$2 M_{r}|| p \|_{L^{t}\left(\mu_{N}(C / K)\right)}$ whenever $p \in P$ and $|z|=r$. Now, there exists $c>0$ such that $\mu_{N} \leq c . \mu$ and therefore $|p(z)| \leq 2 c M_{r} .\|p\|_{L^{t}\left(\mu_{N}(C / K)\right)}$ whenever $p \in P$ and $|z|=r$. By the Maximum Modulus Theorem, $\{z:|z|<r\} \subseteq \operatorname{abpe}\left(P^{t}\left(\mu_{(C / K)}\right)\right.$. Since $\rho<r<1$ is arbitrary, we conclude that $D \subseteq \operatorname{abpe}\left(P^{t}\left(\mu_{(C / K)}\right)\right)$

\section{References}

[1] K. Al-Hami, Weak-Star convergence of measure, International Journal of pure and applied Math. Vol. 88, No. 4, (2013). 